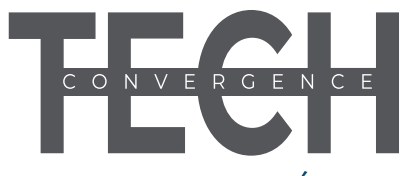

REVISTACIENTÍFICA

Marzo-Agosto 2020

Vol. 4, No. 1,59-68

ISSN 2737-6087

\title{
IMPACTO DE LA INVESTIGACIÓN CUANTITATIVA EN LA ACTUALIDAD
}

\section{Ledys Lisbeth Jiménez González}

\author{
Coordinadora de Investigación del Instituto Superior Tecnológico \\ Particular Sudamericano, Cuenca. \\ investigacionits@sudamericano.edu.ec \\ (iD) https://orcid.org/0000-0001-8743-1206
}

\section{Fecha de recepción:}

Fecha de aceptación:

\section{RESUMEN}

Al asumir el método científico para dar respuesta a las diversas problemáticas que desde las diferentes disciplinas de la ciencia enfrenta el investigador, se hace necesario definir un conjunto de decisiones de orden metodológico que garanticen la rigurosidad científica que exige el proceso investigativo. De tal manera que, ese proceso de toma de decisiones lleva a considerar entre otros componentes el diseño de investigación, el sistema de estrategias a emplear, las tipologías de muestras, los métodos para la recolección de datos, las técnicas para el análisis de resultados, además de los criterios de inclusión y exclusión que garanticen la calidad investigativa en correspondencia con los objetivos previamente establecidos. En la actualidad los diseños de investigación se definen en función de dos grandes paradigmas que fundamentan desde el punto de vista epistemológico los modelos cuantitativo y cualitativo de la investigación. Este ensayo tiene como propósito brindar una orientación didáctica, para quie-

\section{ABSTRACT}

By assuming the scientific method to respond to the various problems faced by the researcher from the different disciplines of science, it is necessary to define a set of methodological decisions that guarantee the scientific rigor required by the investigative process. In such a way that, this decision-making process leads to consider, among other components, nes se inician en el campo de la investigación, sobre los fundamentos del modelo cuantitativo de la investigación científica, su evolución en el tiempo y su pertinencia en el abordaje de problemas que enfrenta la sociedad en la actualidad. De igual manera, se realiza un recorrido conceptual sobre las técnicas e instrumentos del enfoque cuantitativo y su impacto en la generación de grandes avances de la ciencia en las diversas áreas del conocimiento desde sus orígenes hasta la actualidad. Se concluye que la investigación cuantitativa mantiene su impacto y relevancia en los tipos de estudios que requieren de un orden secuencial, riguroso y demostrativo, con una amplia gama de criterios que permita su valoración y aportes a la comunidad científica. La importancia del modelo cuantitativo radica en la aplicación de una postura divergente, manteniendo la objetividad en los fenómenos observados y traducidos en datos numéricos.

Palabras Clave: paradigma, investigación cuantitativa, enfoque

the research design, the system of strategies to be used, the types of samples, the methods for data collection, the techniques for the analysis of results, in addition to the inclusion and exclusion criteria that they guarantee in correspondence with the previously established objectives. At present, research designs are defined based on two major paradigms that are based from the epistemological point of view on the quantitative and qualitative approaches to re- 
search. The purpose of this essay is to provide a didactic orientation, for those who are starting in the field of research, on the foundations of the quantitative model of scientific research, its evolution over time and its relevance in addressing problems facing society nowadays. In the same way, a conceptual tour is made on the techniques and instruments of the quantitative approach and its impact on the generation of great advances in science in various areas of knowledge from its origins to the present. It is concluded that quantitative research maintains its impact and relevance in types of studies that require a sequential, rigorous and demonstrative order, with a wide range of criteria that allow its assessment and contributions to the scientific community. Its importance lies in the application of a divergent position, maintaining objectivity in the observed phenomena and translated into numerical data.

Keywords: paradigm, research, approach, quantitative

Forma sugerida de citar: Jiménez González, L. L. (2020). Impacto de la investigación cuantitativa en la actualidad. Convergence Tech Revista Científica. 4(1), 59-68.

\section{INTRODUCCIÓN}

Al hablar de metodología de la investigación nos referimos al conjunto de decisiones que el investigador debe tomar en función de lograr la rigurosidad científica de la investigación y alcanzar los objetivos que han sido planteados a partir del análisis de una problemática abordada. De tal manera que, ese proceso de toma de decisiones lleva a considerar entre otros componentes el diseño de investigación, sistema de estrategias a emplear, población, tipo de muestras, métodos para la recolección de datos, técnicas para el análisis de resultados, además de los criterios de inclusión y exclusión que garanticen la calidad de la investigación. En la actualidad los diseños de investigación se definen en función de dos grandes paradigmas que se expresan finalmente en el modelo cuantitativo y el modelo cualitativo (Binda \& Benavent, 2013).

El concepto de paradigma puede ser considerado complejo por las múltiples interpretaciones a las que se presta dependiendo del área del conocimiento desde la cual se analiza. No obstante, en la era contemporánea el término es empleado por primera vez en el ámbito de las ciencias por el físico, filósofo e historiador de origen norteamericano Thomas Kuhn (1922-1996), al cual se refería como la totalidad de logros compartidos por una comunidad científica, los cuales son puestos en práctica con el propósito de determinar problemas y buscar soluciones que sean legitimas para los mismos. En su interpretación del concepto de Kuhn, (Hurtado, 1996, 2000) sostiene que:

Tras una teoría científica hay un cierto marco del cual se desarrolla la ciencia, es decir, un paradigma, y este consta de creencias, valores y técnicas que orientan el desempeño de una disciplina científica en un momento determinado. Los científicos que comparte un mismo paradigma están sujetos a las mismas reglas y normas para la práctica científica (p.3).

En este sentido, es correcto afirmar que los sistemas filosóficos como forma de organización del conocimiento a partir de concepciones racionales sobre la realidad, el ser humano o/y el mundo, sientan las bases para el surgimiento de los paradigmas científicos como realizaciones universalmente reconocidas, las cuales durante un periodo de tiempo proporcionan modelos de problemas y soluciones a una comunidad científica. En el análisis sobre la historia de la ciencia Kunh (1962) niega la proveniencia de la evolución de la teoría científica de la estricta acumulación de hechos y afirma que la evolución científica experimentada por la sociedad es producto de un grupo de circunstancias y posibilidades intelectuales sujetas a la transformación y el cambio permanente. No obstante, de acuerdo con (Hurtado, 2000) las revoluciones científicas no se explican cómo fueron entendida por Kuhn, es decir, por el derrocamiento de un paradigma sino con la integración de paradigmas o creación de 
lo que la autora define como de sintagmas. Lo anterior indica que los paradigmas no son estáticos y pueden ser dar origen a nuevos paradigmas en la medida en que la sociedad evoluciona.

De acuerdo (Salas, 2011), la elección por parte de un investigador del modelo de investigación o paradigma de investigación implica el análisis de "elementos epistémicos y de la filosofía de la ciencia tales como los valores epistémicos, los modelos del conocimiento y las leyes científicas y generalizaciones empíricas" (p.14). A continuación, abordaremos los fundamentos del modelo cuantitativo de la investigación científica, su evolución en el tiempo y su pertinencia en el abordaje de problemas que enfrenta la sociedad en la actualidad.

\section{DESARROLLO}

Como es sabido, el ser humano desde su existencia ha estado en búsqueda del conocimiento, y se ha guiado por costumbres, creencias, posturas ancestrales entre otros elementos para conocer y llegar a la raíz de las situaciones que lo rodean la sociedad, llegando a estudiar no sólo las maravillas de la naturaleza sino también el comportamiento social del hombre y los componentes del universo en general. El proceso investigativo tiene como propósito final generar conocimiento a través de la resolución del problema establecido al inicio del estudio (Binda \& Benavent, 2013).

Para (Hernández, R., Fernández, C., \& Baptista, 2010) no es tarea fácil definir con exactitud el momento en que se da inició al enfoque cuantitativo, no obstante, el análisis de diferentes fuentes y su evolución se ha sostenido en el tiempo; "algunos autores de la historia de la ciencia los ubican desde Aristóteles y otros en diferentes puntos de los siglos XVI, XVII y XVIII" (p.8).

En palabras de(Gorbea-Portal, 2006), el paradigma cuantitativo tiene sus orígenes en el pensamiento científico de era moderna, es fortalecido por el positivismo lógico y tiene como propósito la búsqueda de la objetividad científica de los hechos o causas sociales en función de la explicación (Erkärunge) de la verdad sobre la base de la medición penetrante, fundamentada en el control y la valoración de parámetros estrictamente objetivos que por supuesto anula por completo los prejuicios y toda po- sibilidad de elementos de tipo subjetivos.

Para el alcance de este propósito sus fundadores generaron un proceso de emulación de los métodos de las ciencias naturales, por lo cual es considerado por mucho tiempo como un paradigma con preeminencia en las ciencias naturales y exactas.

El desarrollo de estos procedimientos y la consecuente exactitud rigurosa de que hacen gala se convierten en la piedra filosofal que ha de deslumbrar a los científicos sociales que pretenden desprenderse de todo prejuicio y valoración subjetiva. Ya Comte fijó esta exigencia como requisito indispensable para el alumbramiento de esa "ciencia» de la que él fue digno fundador: la sociología. Pues bien, nos encontramos aquí con el primer error: el de tomar el método de las ciencias de la naturaleza. Y el error estriba precisamente en que la relación entre el investigador y lo investigado es radicalmente distinta en dichas ciencias y en las sociales. Mientras las primeras se enfrentan con el objeto como algo que está ahí, que está dado, que es ajeno al sujeto, en el saber social el sujeto es también objeto, el investigador forma parte de lo investigado(Fernández Navas, M., \& Postigo-Fuentes, 2020)

En este sentido, (Sarduy, 2007) coincide en que la producción bibliográfica cuantitativa se encuentra orientada principalmente al desarrollo de estudios cuyo propósito es la exposición de datos y descripciones de la realidad social, dejando relegados los estudios que promuevan explicaciones sobre esa realidad social.

El positivismo marcó en su momento un gran auge influenciado por las ciencias exactas, tales como: la química, biología, física entre los que han destacado los más importantes científicos como Galileo, Isaac Newton, Nicolás Copérnico, Charles Darwin, quienes basaron sus estudios en la objetividad, medición, manipulación de variables que permitían la verificación del fenómeno estudiado (GorbeaPortal, 2006 p.3).

De tal manera que, el enfoque cuantitativo en las ciencias sociales tiene su génesis precisamente en la obra de Auguste Comte (1798-1857) y Émile Durkheim 
(1858-1917), con la influencia significativa de Francis Bacon (1561-1626), John Locke (1632-1704) e Immanuel Kant (1724-1804). Por lo cual, se fundamenta en los parámetros del positivismo (Hernández, R., Fernández, C., \& Baptista, 2010).

No obstante, a finales del siglo XIX el positivismo comienza a ser reemplazado por una nueva corriente denominada el "pospositivismo", el cual tiene sus bases en el paradigma antecesor pero con un carácter más abierto y flexible. Según (Hernández, R., Fernández, C., \& Baptista, 2010 p.3).

Tal visión comienza a gestarse hacia fines del siglo XIX, pero realmente se consolida hacia la mitad del siglo pasado. La germinación la inician las obras de autores como Wilhelm Dilthey (1833-1911) y William James (1842-1910). Karl Popper (19021994) lo impulsa de forma notoria. Además, en cada campo o disciplina, diversos autores lo desarrollan.

La diferencia entre ambas corrientes del pensamiento radica en la concepción del conocimiento. El pospositivismo supera la visión de la percepción como un simple reflejo de las cosas reales y el conocimiento como copia de la realidad. El conocimiento es para el pospositivismo el resultado de la interacción directica entre el investigador y el objeto de estudio.

El pospositivismo como nuevo sistema de organización del conocimiento acoge al enfoque cuantitativo y asigna las siguientes características:

1 Recolectar datos en la forma de puntuaciones (que se origina en las matemáticas). Es decir, los atributos de fenómenos, objetos, animales, personas, organizaciones y colectividades mayores son medidos y ubicados numéricamente.

2 Analizar tales datos numéricos en términos de su variación.

3 La esencia del análisis implica comparar grupos o relacionar factores sobre tales atributos mediante técnicas estadísticas (en el caso de las ciencias del comportamiento, mediante experimentos y estudios causales o correlacionales)(Hernández, R., Fernández, C., \& Baptista, 2010).
En la actualidad la investigación con enfoque cuantitativo y su impacto en el terreno del campo investigativo basado en el empirismo, es decir, en la experiencia, observación y análisis de los hechos, los cuales son procesados mediante diversas técnicas numéricas para la obtención y fiabilidad en sus resultados. Es evidente que las técnicas e instrumentos del enfoque cuantitativo son una herramienta valiosa y de gran utilidad que han generado mediante su uso importantes avances a la ciencia en muchas áreas del conocimiento. Desde el punto de vista de (Sarduy, 2007 p.8), la aplicación del enfoque cuantitativo en investigación permite:

Recoger, procesar y analizar datos cuantitativos o numéricos sobre variables previamente determinadas. Esto ya lo hace darle una connotación que va más allá de un mero listado de datos organizados como resultado; pues estos datos que se muestran en el informe final, están en total consonancia con las variables que se declararon desde el principio y los resultados obtenidos van a brindar una realidad específica a la que estos están sujetos (p8).

Por otra parte, la investigación cuantitativa, posee algunos elementos claves que la diferencian de otro tipo de investigaciones, precisamente radican en su propósito, mediante la obtención de datos numéricos a partir de la predicción de fenómenos abordados. Para tales fines, hace uso de un diseño y método estructurado en la cual requiere de la intervención y manipulación de la o las variables por parte de quien investiga y puede ser descriptiva, correlacional, experimental, comparativa o causal. Aplicando algunas estrategias de recolección de datos como: encuestas, cuestionarios, entrevistas, observación, observación estructurada, muestreo probabilístico, revisión de documentos, test. Y finalmente aplica la interpretación de dichos datos con certeza y objetividad (Sarduy, 2007).

Diversas son las estrategias para la recolección de datos en la investigación, no obstante, los arriba mencionados están entre lo más comunes en dicho proceso, que además permite al investigador obtener resultados de forma exhaustiva y detallada, los cuales podrán ser comprendidos al ser representado en cuadros y gráficos. 
Figura 1 » En la figura se ilustra algunas de las estrategias para la recolección de datos más usados en la investigación Cuantitativa. Elaboración propia.

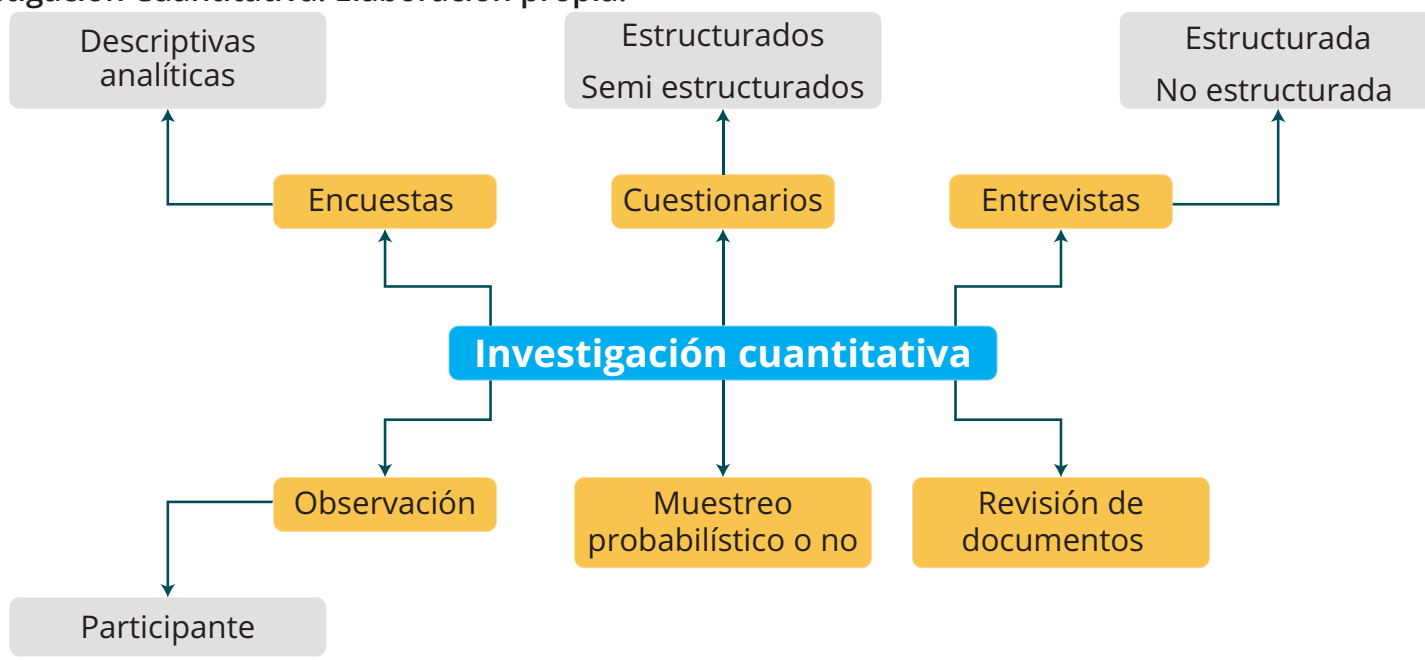

Tal como se representa en la figura $\mathrm{N}^{\circ} 1$, en la investigación cuantitativa se vale de diversas estrategias para obtener la información, facilitándole al investigador el enriquecimiento de su trabajo. Su elección va a depender de la naturaleza del mismo. Ahora bien, se procede a revisar la argumentación de algunos autores en cuanto a la definición de los métodos arriba señalados.

La encuesta, según (Tamayo y Tamayo, 2003 p.32) "es aquella que permite dar respuestas a problemas en términos descriptivos como de relación de variables, tras la recogida sistemática de información según un diseño previamente establecido que asegure el rigor de la información obtenida".

Al respecto, (Arias, 2012 p.72) señala "es una técnica que pretende obtener información que suministra un grupo o muestra de sujetos acerca de si mismos, o en relación con un tema en particular".

Cabe mencionar, además, que la encuesta tiene algunos propósitos interesantes de los cuales se beneficia la investigación cuantitativa en su proceso. Al respecto, (Kerlinger, 1997) menciona los siguientes:

- Complementación de otros métodos de investigación.

- Ser el principal instrumento de la investigación

- Servir de instrumento exploratorio

Por lo tanto, la encuesta como técnica es una herramienta valiosa para la recolección de información inhe- rente a una temática en particular. Dependiendo de la información que se requiera puede ser de tipo descriptiva, en la cual su función está centrada precisamente en recabar, documentar y describir el estado actual en el que se encuentra la población determinada en el momento de aplicar la misma. Por otro lado, se tiene la analítica, que además de describir busca el por qué en una situación específica. Se pueden aplicar desde diversos medios, tales como: impreso, telefónica, dispositivos móviles, web.

Entre otras de las herramientas valiosas, está el instrumento del cuestionario. Para (Arias, 2012 p.75) consiste en "la modalidad de encuesta que se realiza de forma escrita mediante un instrumento o formato en papel contentivo de una serie de preguntas. Se le denomina cuestionario autoadministrado porque debe ser llenado por el encuestado, sin intervención del encuestador".

Los cuestionarios, suelen clasificarse de la siguiente manera de acuerdo con la redacción de las preguntas y su presentación dependerá del medio en el cual el investigador seleccione, bien sea impreso, en hojas de papel o electrónico, haciendo uso de APP Aplicaciones mediante dispositivos:

- Cuestionarios estructurados: en este tipo de cuestionarios las preguntas o posibles respuestas son estandarizadas.

- Cuestionarios semi estructurados: se caracterizan por presentar un orden que no es estricto, en sus preguntas y lógicamente en sus posibles res- 
puestas, acá se pone en manifiesto la existencia de un patrón con sus enunciados.

- Cuestionarios no estructurados: en este tipo de cuestionario, la persona a quien va dirigido, tendrá más libertad en responder las preguntas, ya que, son preguntas son redactadas de manera general y éstas no siguen un orden en especial.

Por otro lado, tanto en las investigaciones de corte cualitativo o cuantitativo se puede hacer uso de la técnica de la observación, sin embargo, la investigación cuantitativa, se caracteriza por obtener información presentada estadísticamente a diferencia de la cualitativa, que suelen ser más largas y con muestras pequeñas y representadas de manera descriptiva, o en su defecto sería un estudio complementario.

La observación, según (Mendez, 1995 p. 98) se define como un "proceso sistemático que permite detectar particularidades dentro de un proceso o contexto determinado. La observación es también un proceso empleado en diversos campos del quehacer investigativo". Para (Arias, 2012 p.70) la observación "es una técnica que consiste en visualizar o captar mediante la vista, en forma sistemática, cualquier hecho, fenómeno o situación que se produzca en la naturaleza o en la sociedad, en función de unos objetivos de investigación preestablecidos". De tal modo que, la observación es la acción organizada en la cual se percibe o capta la realidad abordada. En tal sentido, mediante la técnica de la observación, el investigador puede o no formar parte activa de la realidad observada.

En el caso, que el investigador se involucre en el grupo observado, se estará en presencia de la denominada observación participante. Caso contrario, cuando quien investiga no se involucra con el grupo observado, se llama observación no participante. La observación participante, queda claro que el investigador se involucra con el grupo a observar, es decir, son sujetos y objetos activos en el estudio, comparten vivencias, diversas actividades que le permiten enriquecer y llevar registros a quien investigan y es fundamental en los estudios de índole cualitativos.

Por lo contario, en la observación no participante el investigador es un miembro externo, no se involucra y se mantiene al margen del fenómeno estudiado, asume una posición pasiva, limitándose solo a tomar registros de lo manifestado frente a él, con el propósito de darle la mayor veracidad al proceso abordado. Sin embargo, se pueden ubicar dos aspectos interesantes en este tipo de observación: una directa o indirecta. En la primera, el investigador observa desde el escenario estudiado, pero sin relacionarse con el grupo; y la segunda, éste no se encuentra en el espacio de estudio del fenómeno, es decir, no se encuentra dentro del espacio para observar, sino que centra su atención en la observación de fuentes documentales, fotográficas, audiovisuales.

En este sentido, la investigación cuantitativa se beneficia en gran medida con el uso de ésta valiosa técnica de registro como fuente de documentación, la cual enriquece en gran medida el estudio mediante el suministro de datos que son representados de forma estadística manteniendo la mayor suma de objetividad posible.

Ahora bien, otra de las herramientas empleadas en la investigación cuantitativa es la entrevista. De acuerdo con (Arias, 2012), la entrevista "más que un simple interrogatorio, es una técnica basada en un diálogo o conversación -cara a cara-, entre el entrevistador y el entrevistado acerca de un tema previamente determinado, de tal manera que el entrevistador pueda obtener la información requerida". (p.74). Sin embargo, en los estudios cuantitativos esta técnica posee una particularidad, la cual radica en que a todas las personas a la cuales se le aplica esta técnica se les harán el mismo tipo de preguntas y por ende se obtendrá el mismo tipo de respuestas.

La entrevista se clasifica en:

- Entrevista estructurada o formal: se realiza a partir de una guía prediseñada, la cual contiene las preguntas que serán formuladas al entrevistado. De ser necesario, la misma guía de entrevista puede servir como instrumento para registrar las respuestas, aunque también otros instrumentos para el registro de la información, como por ejemplo: un grabador, una cámara de video, celulares, entre otros.

- Entrevista no estructurada o informal: para su aplicación el investigador no dispone de una guía de preguntas previamente elaboradas. No obstante, se desarrolla en función de los objetivos planteados en el proyecto de investigación, los 
cuales permiten definir el tema de la entrevista. Se requiere habilidades y destrezas por parte del entrevistador para llevar la secuencia de las interrogantes planteadas.

- Entrevista semi-estructurada: en este caso, puede disponerse de una guía de preguntas pero el entrevistador puede realizar preguntas adicionales, de manera extraordinaria, es decir que no estén establecidas en la guía. El carácter de esta modalidad de entrevista es su flexibilidad. El entrevistador dispone de los instrumentos específicos contentivos de las interrogantes y puede emplear instrumentos otros instrumentos como la grabadora o la cámara de video o el celular, entre otros.

Continuando con la precisión de algunas estrategias más usadas en la investigación cuantitativa, según lo graficado en la figura $\mathrm{N}^{\circ} 1$, finalmente se encuentra el muestreo probabilístico y la revisión de documentos. En relación con el muestreo (López-Roldán \& Fachelli, 2015) expresan que "el objetivo general de todo muestreo es llegar a conocer determinadas características de una población, a partir de una selección de unidades de ésta, con el menor coste posible en dinero, tiempo y trabajo" (p.12). En el muestreo probabilístico, "cada elemento del universo tiene una probabilidad conocida y no nula de figurar en la muestra, es decir, todos los elementos del universo pueden formar parte de la muestra". Es decir, que cada elemento que forma parte del universo tendrá la posibilidad de ser elegido para ser considerado en la muestra. De acuerdo con (López-Roldán \& Fachelli, 2015) en esta categoría es posible distinguir entre:

- Muestreo aleatorio simple

- Muestreo sistemático

- Muestreo estratificado

- Muestreo por conglomerados

- Muestreo polietápico

A diferencia del muestreo probabilístico, en el muestreo no probabilístico los encuestados no se conocen las probabilidades de cada unidad de muestreo de pertenecer a la muestra (López-Roldán \& Fachelli, 2015). Destacan en esta categoría los siguientes tipos:

- Muestreo por cuotas

- Muestreo casual o incidental
- Muestreo de conveniencia

- Muestreo intencional o razonado

- Muestreo de bola de nieve

Es evidente las diferencias entre ambos tipos de muestreo en las cuales se refleja que, a diferencia del muestreo probabilístico, en la cual los individuos que forman parte de la población, son seleccionados de manera aleatoria, teniendo la oportunidad de ser parte de la muestra, mientras que en el no probabilístico es tomado a criterio y por tanto hace parte la subjetividad del investigador y no toda la población tendrá la misma oportunidad de ser parte de la muestra.

Ahora bien, la última estrategia que abordaremos en este ensayo es la revisión documental, considerada una de las más empleadas en la investigación cuantitativa. A juicio de (Hurtado, 2008) la revisión documental consiste en la técnica para recogida de información de forma escrita sobre un tema determinado con el objeto de identificar variables que se relacionan indirecta o directamente con el tema establecido. Ciertamente, esta técnica sirve de apoyo en la investigación cuantitativa, permitiéndole al investigador la revisión de investigaciones ya existentes inherentes a su temática y establecer en función de ello metas de mayor proyección en su trabajo investigativo.

A propósito, el impacto de la investigación cuantitativa se encuentra fuertemente vinculado con la riqueza que le genera el uso de las diversas técnicas e instrumentos para la recolección de información y lógicamente su procesamiento estadístico, en virtud de la fiabilidad obtenidos en los resultados.

Por último, consideramos necesario exponer algunas fases que se deben tener en cuenta para el abordaje de una investigación cuantitativa, según (Hernández Sampieri et al., 2010):

Fase 1: la idea: en esta fase el investigador ordena sus inquietudes, intereses y motivaciones y luego las sistematiza, para constituirse en los que se denomina el punto de partida.

Fase 2: planteamiento del problema: el investigador ya teniendo el punto de partida para dar inicio a su investigación, requiere delimitar el estudio a abordar, dejando establecida de manera clara y precisa qué es lo que desea estudiar. 
Fase 3: revisión de la literatura y desarrollo del marco teórico: el investigador hace revisión bibliográfica de todo aquel material que sirva para enriquecer su estudio, teorías, paradigmas que le sirvan para argumentar lo que pretende investigar. Plantea la base de la fundamentación conceptual y formulación del marco teórico.

Fase 4: visualización del alcance de estudio: en este momento el investigador debe identificar con mucha precisión hasta donde será dicho alcance y a qué resultados pretende obtener, antes de dar inicio a la investigación.

Fase 5: definición de hipótesis y definición de variables: el investigador a partir de la elaboración de las hipótesis podrá anticipar, planteará un acercamiento al intentar explicar el fenómeno en estudio con posibles respuestas.

Fase 6: desarrollo del diseño de investigación: en esta fase el investigador seleccionará los métodos y estrategias que respondan a sus preguntas que orientarán su estudio de investigación.

Fase 7: definición y selección de la muestra: el inves- tigador en esta fase definirá la muestra, es decir, el grupo sub grupo representativo de la población, bien sea probabilística o no probabilística.

Fase 8: recolección de datos: en esta fase hará usos de diversas herramientas tales como técnicas e instrumentos de recolección de información. Aplicará los previamente ya establecidos en la metodología.

Fase 9: análisis de los datos: una vez ya finalizada con la fase anterior, de recolección de información, el investigador realizará el análisis y procesamiento de forma organizada y coherente a fin de fin de descifrar y sacar sus relaciones.

Fase 10: elaboración del reporte de resultados: en esta última fase, se recomienda que el investigador establezca de qué forma va a presentar los resultados y que tipo de reporte. La creatividad del investigador se pone de manifiesto en esta fase, ya que puede presentar diversos elementos gráficos que representen los resultados, además de aplicar las normativas de presentación de trabajos bien seas normas APA, ACS Style, Chicago Style, entre otras.

Figura 2 » Fases de la investigación cuantitativa. Basado en Sampieri, H (2010). Elaboración propia

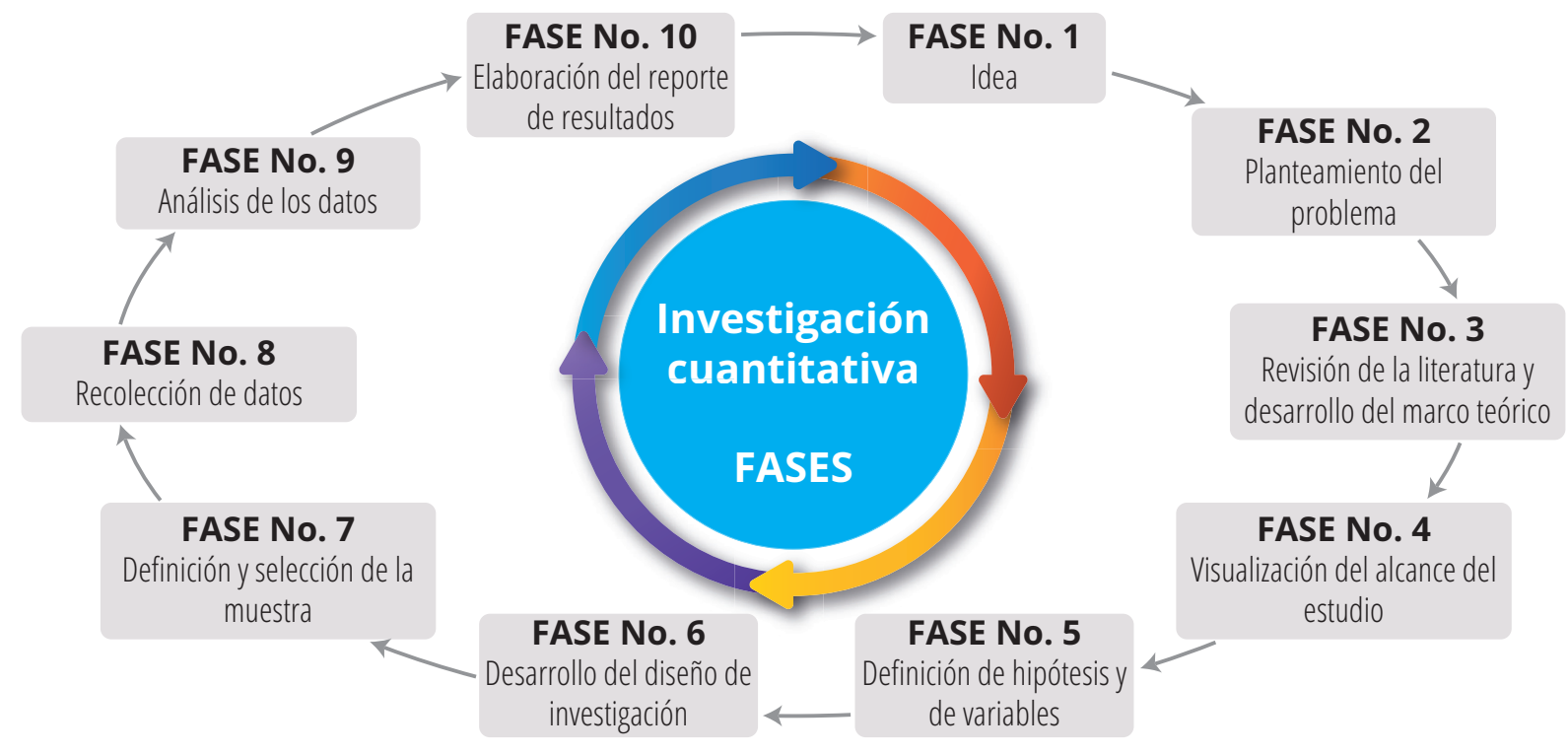

Una vez realizado el recorrido de las fases presentes en la investigación cuantitativa, se manifiesta claramente, que en este tipo de estudios permite al investigador llevar un proceso metódico, riguroso y exhaustivo en cuanto a la medición, interpretación, procesamiento y análisis estadísticos de los datos. 


\section{CONCLUSIONES}

La investigación cuantitativa fundamentada en el paradigma pospositivista no ha sido remplazada por el enfoque cualitativo, cada tipo de investigación como bien se abordó en este ensayo, poseen atributos particulares y elementos característicos que los diferencian uno del otro.

La investigación cuantitativa mantiene su impacto y relevancia en tipos de estudios que requieren de un orden secuencial, riguroso y demostrativo, con una amplia gama de criterios que permiten su valoración y aportes a la comunidad científica. Su importancia, radica en la aplicación de una postura divergente, manteniendo la objetividad en los fenómenos observados y traducidos en datos numéricos.

En el desarrollo de sus procesos imperan los análisis de tipo deductivos, es decir, que va de lo general a lo particular, donde la recolección de datos para luego probar hipótesis ya establecidas que le permitan comprobar las mismas, basadas en el conteo, la medición estadística para relacionar con exactitud ciertos patrones de comportamientos presentes en una población.

Ciertamente, se caracteriza por hacer generalizaciones a partir los resultados obtenidos. En consecuencia, este tipo de enfoque, basado en el empirismo devela su impacto justo en la objetividad de sus estudios, en medir y calcular los determinados comportamientos de un fenómeno para luego hacer las generalizaciones correspondientes a partir de lo ya probado.

\section{BIBLIOGRAFÍA}

Arias, F. G. (2012). El Proyecto de Investigación. Introducción a la Metodología Científica. 6ta ... - Fidias G. Arias - Google Libros. https://books. google.es/books?hl=es\&lr=\&id=W5nOBgAAQBA J\&oi $=$ fnd \&pg $=P A 11 \& d q=$ fidias + arias + pro yecto\&ots $=k Y j J c s q w o 3 \& s i g=1 S z R 5 M X N R-$ NU918KjhHz1y97riZo\#v=onepage\&q=fidias arias proyecto\&f $=$ false

Binda, N. U., \& Benavent, F. B. (2013). InvestIgacIón cuantItatIva e InvestIgacIón cualitatIva: buscando las ventajas de las diferentes metodologías de InvestIgaclón. Revistas.Ucr.Ac.Cr. https://revistas.ucr.ac.cr/index.php/economicas/article/ view/12730
Fernández Navas, M., \& Postigo-Fuentes, A. Y. (2020). Vista de La situación de la investigación cualitativa en Educación: ¿Guerra de paradigmas de nuevo? | Márgenes Revista de Educación de la Universidad de Málaga. https://www.revistas.uma.es/index. php/mgn/article/view/7396/7054

Gorbea-Portal, S. (2006). Trascendencia del paradigma cuantitativo en la investigación bibliotecológica y de la información. http://www. scielo.org.mx/scielo.php?script=sci_arttext\&pi$\mathrm{d}=$ S0187-358X2006000200001

Hernández, R., Fernández, C., \& Baptista, M. (2010). HISTORIA DE LOS ENFOQUES CUANTITATIVO, CUALITATIVO Y MIXTO: RAIICES Y MOMENTOS DECISIVOS INTRODUCCIÓN.

Hernández Sampieri, R., Fernández Collado, C., \& del Pilar Baptista Lucio, M. (2010). Metodología de la investigación, 5ta Ed. www.FreeLibros.com

Hurtado, Y. (2008). Tipos de investigacion. https:// www.google.com/search?ei=RacZYPXMBImd5g KH26XACA\&q=yaqueine+hurtado++tipos+de+investigacion\&oq=yaqueine+hurtado++tipos+de+investigacion\&gs_lcp=CgZwc3ktYWIQAzIECAAQDTIICAAQCBANEB4yCAgAEAgQDRAeOgIIADoGCAAQCBAeOggIABAIEAcQHlDtmQFYhtABYJTSAWgCcAB4AoABk

J, H. B. (2000). Metodología de la investigación holística. Sidalc.Net. http://www.sidalc.net/cgi-bin/wxis. exe/?IsisScript=catalco.xis\&method=post\&formato $=2 \&$ cantidad $=1$ \&expresion $=m f n=065384$

Kerlinger, F. (1997). Kerlinger, F. (1997). Investigación del comportamiento. México, D.F.: McGraw-Hill. - Buscar con Google. https://www.google.com/ search?q=Kerlinger\%2C+F.+(1997).+Investigación+del+comportamiento.+México\%2C+D.F.\%3A+McGraw-Hill.\&oq=Kerlinger\%2C+F.+(1997).+Investigación+del+comportamiento.+México\%2C+D.F.\%3A+McGraw-Hill.\&aqs=chrome..69i57. 327j0j7\&sourceid=chrome\&

López-Roldán, P., \& Fachelli, S. (2015). METODOLOGÍA DE LA INVESTIGACIÓN SOCIAL CUANTITATIVA.

Mendez Alvarez, C. E. (1995). Metodología: Guía para elaborar de Diseños de investigación en ciencias economicas, contables y administrativas. 2a. Edicion. Metodología: Guía Para Elaborar de Diseños de Investigación En Ciencias Economicas, Contables y Administrativas. https://repository.urosario.edu. co/handle/10336/30059 
Salas, H. (2011). Investigación Cuantitativa (Monismo Metodológico) y Cualitativa (Dualismo Metodológico). In Cinta moebio (Vol. 40). www. moebio.uchile.cl/40/salas.html

Sarduy Domínguez, Y. (2007). El análisis de información y las investigaciones cuantitativa y cualitativa. http://scielo.sld.cu/scielo.php?script=sci_arttext\&pid=S0864-34662007000300020

Tamayo y Tamayo, M. (2003). Tamayo y Tamayo, Mario - El Proceso de la Investigación Científica | Conocimiento | Epistemología. https://es.scribd. com/doc/12235974/Tamayo-y-Tamayo-Mario-ElProceso-de-la-Investigacion-Cientifica 